\title{
On the Irregularity of Special Non-Canonical Surfaces
}

By

\author{
Kazuhiro KonNo *
}

\begin{abstract}
We consider minimal surfaces of general type whose canonical map is "special" meaning that it is composed of a pencil or its degree is high. We characterize, to some extent, Beauville's examples of irregularity 2 in the pencil case, and show that the irregularity is at most 12 when the canonical degree is 5 .
\end{abstract}

\section{Introduction}

Let $S$ be a minimal surface of general type defined over $C$, and let $K=K_{S}$ denote a canonical divisor. If $p_{g}>1$, we can consider the rational map associated with $|K|$, the canonical map $\Phi_{K}: S \rightarrow P^{p_{g}-1}$. We put $\Sigma=\Phi_{K}(S)$ and let $\phi_{K}: S \rightarrow \Sigma$ be the induced rational map. When $\phi_{K}$ is not birational, some important results were obtained by Beauville and Xiao :

(1) Suppose that $\Sigma$ is a curve, that is, $|K|$ is composed of a pencil. We get a relatively minimal fibration $f: X \rightarrow B$ after blowing up the base points and taking the Stein factorization if necessary. Put $b=g(B)$ and let $g$ be the genus of a general fibre of $f$. Beauville [1] showed that $g \leq 5$ when $p_{g}$ is large. Later, Xiao [12] showed that either $b=q=1$ or $b=0, q \leq 2$.

(2) Suppose that $\Sigma$ is a surface. It is well-known that $\Sigma$ is a ruled surface when its degree is small (cf. [1], [14] or [10]). Hence, if $d_{c a n}:=\operatorname{deg} \phi_{K}$ is large, Miyaoka-Yau's inequality implies that $\Sigma$ is ruled and, as in the previous case, $S$ has a pencil of curves of genus $g$ induced by the ruling of $\Sigma$. Beauville [1] showed that $d_{c a n} \leq 9$ when $p_{g}$ is large enough. Xiao showed that $d_{c a n}=9$ is actually impossible for $p_{g}>132$ ([14]), and that $q \leq 3$ when $d_{c a n} \geq 7, p_{g}>115$ ([14] and [16]). He also

\footnotetext{
Communicated by S. Mori, July 21, 1993.
}

Supported in part by a Grant under The Monbusho International Scientific Research Program : 04044081

1991 Mathematics Subject Classification : 14J29

* Department of Mathematics, Faculty of Science, Osaka University, Toyonaka, Osaka 560, Japan. 
proved that there is a bound on $q, g$ when $d_{c a n}=5,6$. After that, Sun [11] has shown $q \leq 5$ when $d_{c a n}=6$ and $p_{g}>55$, along an analogous line.

The purpose of this article is to give a slight refinement of the above results. Our main interest is in the cases $q=2$ in (1) and $d_{c a n}=5$ in (2). We show that a surface with $q=2$ whose canonical map is composed of a pencil is essentially an example of Beauville $[1,2.5]$ when the Albanese map is not surjective (Theorem 3.6), and that $q \leq 12$ if the canonical map is of degree 5 onto the image (Theorem 4.5). As one may learn from (1) and (2), we are naturally led to studying fibred surfaces $f: X \rightarrow B$. We use the powerful methods due to Xiao in order to analize $f_{*} \omega_{X}$. Hence the paper should be regarded as an appendix to his remarkable papers, especially to [14].

\section{$\S 1$. Irregularity of Fibred Surfaces}

In this and the next sections, we recast Xiao's method in [14] and prepare some results for the later use. See also $[12],[15],[16],[1],[3]$ and $[9]$.

1.1. Let $\mathscr{E}$ be a locally free sheaf on a non-singular projective curve $B$. We put $\mathscr{E}^{*}=\operatorname{Hom}\left(\mathscr{E}, \omega_{B}\right)$ and $\mu(\mathscr{E})=\operatorname{deg}(\mathscr{E}) / \mathrm{rk}(\mathscr{E})$. According to [4], we have a filtration of $\mathscr{E}$ by locally free subsheaves $\mathscr{E}_{i}$ :

$$
0=\mathscr{E}_{0} \subset \mathscr{E}_{1} \subset \cdots \subset \mathscr{E}_{l}=\mathscr{E}
$$

which satisfies

(i) $\mathscr{E}_{l} / \mathscr{E}_{i-1}$ is semi-stable,

(ii) $\mu_{i}(\mathscr{E})>\mu_{i+1}(\mathscr{E})$, where $\mu_{i}(\mathscr{E}):=\mu\left(\mathscr{E}_{i} / \mathscr{E}_{i-1}\right)$.

As usual, we call such a filtration the Harder-Narashimhan filtration of $\mathscr{E}$. Note that we have

$$
(\operatorname{rk}(\mathscr{E})-1) \mu_{1}(\mathscr{E})+\mu_{l}(\mathscr{E}) \geq \operatorname{deg}(\mathscr{E})
$$

Let $\pi: \mathbb{P}(\mathscr{E}) \rightarrow B$ be the associated projective bundle. We denote by $H(\mathscr{E})$ and $F$ a (relatively ample) tautological divisor and a fibre of $\pi$, respectively. The locally free sheaf $\mathscr{E}$ is called nef if and only if $H(\mathscr{E})$ is nef. By [9], the $Q$-divisors $H(\mathscr{E})$ $-\mu_{l}(\mathscr{E}) F$ and $H(\mathscr{E})-\mu_{1}(\mathscr{E}) F$ are respectively nef and pseudo-effective.

1.2. Let $f: X \rightarrow B$ be a relatively minimal fibration of non-singular projective surface $X$ onto a non-singular projective curve $B$ of genus $b$. We assume that $X$ is of general type and $p_{g}>0$. We let $g$ denote the genus of a general fibre $D$ of $f$. Then $g \geq 2$. By Arakelov's theorem [2], the relative dualizing sheaf $\omega_{X / B}$ is nef. By [3], $f_{*} \omega_{X}$ is a direct sum of a locally free sheaf and $q-b$ copies of $\omega_{B}$, and $f_{*} \omega_{X / B}=$ 
$f_{*} \omega_{X} \otimes \omega_{B}^{-1}$ is nef.

From now on, we let $\mathscr{E}$ be the locally free subsheaf of $f_{*} \omega_{X}$ generically generated by elements in $H^{0}\left(f_{*} \omega_{X}\right)$; the quotient $\mathscr{E}^{\prime}=f_{*} \omega_{X} / \mathscr{E}$ is also locally free. Put $r=\operatorname{rk}(\mathscr{E})$ and let $0 \subset \mathscr{E}_{1} \subset \cdots \subset \mathscr{E}_{l}=\mathscr{E}$ be the Harder-Narashimhan filtration for $\mathscr{E}$.

For each $i$, the natural sheaf homomorphism $f^{*} \mathscr{E}_{i} \rightarrow f^{*} f_{*} \omega_{X} \rightarrow \omega_{X}$ induces a rational map $\phi_{i}: X \rightarrow \boldsymbol{P}\left(\mathscr{E}_{1}\right)$. Let $\rho_{i}: X_{i} \rightarrow X$ be the elimination of the indeterminacy of $\phi_{i}$, and let $\widetilde{\phi}_{1}: X_{i} \rightarrow \boldsymbol{P}\left(\mathscr{E}_{l}\right)$ be the induced holomorphic map. We denote by $\tilde{M}_{i}$ the pull-back to $X_{i}$ of $H\left(\mathscr{E}_{\imath}\right)$ via $\widetilde{\phi}_{i}$. Then $\tilde{M}_{i}-\mu_{i}(\mathscr{E}) \rho_{i}^{*} D$ is nef, since so is $H\left(\mathscr{E}_{i}\right)-$ $\mu_{i}(\mathscr{E}) F$. Put $M\left(\mathscr{E}_{i}\right)=\left(\rho_{l}\right)_{*} \tilde{M}_{l}$. Then $M\left(\mathscr{E}_{l}\right)-\mu_{i}(\mathscr{E}) D$ is nef. Note that $K_{X} \equiv$ $M\left(\mathscr{E}_{l}\right)+Z\left(\mathscr{E}_{l}\right)$ with an effective divisor $Z\left(\mathscr{E}_{l}\right)$, where $\equiv$ denotes the numerical equivalence.

Let $\mathscr{F}^{\prime}$ be the locally free subsheaf of $\mathscr{E}^{*}$ generated by $H^{0}\left(\mathscr{E}^{*}\right)$, and put $\mathscr{F}=$ $\left(\mathscr{F}^{\prime}\right)^{*}$. Then $\mathscr{F}$ and $\mathscr{F}^{*}$ are both nef. We have $p_{g}=h^{0}\left(f_{*} \omega_{X}\right)=h^{0}(\mathscr{E})$ and $h^{1}(\mathscr{E})$ $=h^{1}(\mathscr{F})=h^{0}\left(\mathscr{F}^{*}\right)$ by the choice of $\mathscr{E}$ and $\mathscr{F}$.

Proposition 1.3. With the above notation,

$$
q(X) \leq b+\operatorname{rk}(\mathscr{F})-(b-1)(g-r)
$$

If the equality holds here, then $\operatorname{deg}\left(\mathscr{E}^{\prime}\right)=2(b-1)(g-r)$ and $\mathscr{F}$ is a direct sum of $\mathrm{rk}(\mathscr{F})$ copies of $\omega_{B}$.

Proof. The following inequalities were shown in $[14$, p. 477$]$ :

$$
\begin{aligned}
h^{1}(\mathscr{E})=h^{1}(\mathscr{F}) & \leq b \operatorname{rk}(\mathscr{F})-\frac{1}{2} \operatorname{deg}(\mathscr{F}), \\
\operatorname{deg}\left(\mathscr{E}^{\prime}\right) & \geq 2(b-1)(g-r) . \\
\operatorname{deg}(\mathscr{F})+\operatorname{deg}\left(\mathscr{E}^{\prime}\right) & \geq 2(b-1)(g-r+\operatorname{rk}(\mathscr{F})),
\end{aligned}
$$

We have an exact sequence

$$
0 \rightarrow \mathscr{E} \rightarrow f_{*} \omega_{X} \rightarrow \mathscr{E}^{\prime} \rightarrow 0
$$

and $h^{0}(\mathscr{E})=h^{0}\left(f_{*} \omega_{X}\right)=p_{g}$. Since we have $h^{1}\left(f_{*} \omega_{X}\right)=q(X)-b$ by $[3$, Theorem (3.1)], we get $q-b=h^{1}(\mathscr{E})-\chi\left(\mathscr{E}^{\prime}\right)$. By the Riemann-Roch theorem and (1.3), we have $\chi\left(\mathscr{E}^{\prime}\right)=\operatorname{deg}\left(\mathscr{E}^{\prime}\right)-(b-1)(g-r) \geq \operatorname{deg}\left(\mathscr{E}^{\prime}\right) / 2$. Applying (1.2), we get $q-b \leq b \operatorname{rk}(\mathscr{F})-\left(\operatorname{deg}(\mathscr{F})+\operatorname{deg}\left(\mathscr{E}^{\prime}\right)\right) / 2$. Hence the inequality follows from (1.4). If the equality holds there, then the equalities hold in (1.2), (1.3) and (1.4). Hence we have $\operatorname{deg}\left(\mathscr{E}^{\prime}\right)=2(b-1)(g-r), \operatorname{deg}(\mathscr{F})=2(b-1) \operatorname{rk}(\mathscr{F})$ and $h^{1}(\mathscr{F})=\operatorname{rk}(\mathscr{F})$. Since $\mathscr{F}$ and $\mathscr{F}^{*}$ are both nef, we see that $\mathscr{F}^{*}$ is semi-stable of degree 0 and $h^{0}\left(\mathscr{F}^{*}\right)=\operatorname{rk}(\mathscr{F})$. Since $\mathscr{F}^{*}$ is generated by its global sections, it is 
a direct sum of $\mathcal{O}_{B}$.

Q. E. D.

Corollary 1.4. Assume that $\operatorname{rk}(\mathscr{F})=r$. Then $p_{g} \leq b r$. Furthermore, $f: X \rightarrow B$ is locally trivial if (and only if) the equality holds in (1.4). In particular, when $q=b$ $+r-(b-1)(g-r), f$ is locally trivial and $\mathscr{E} \simeq \omega_{B}^{\oplus r}$.

Proof. Since rk $(\mathscr{F})=r$, we have $\mathscr{F}=\mathscr{E}$. Hence $\mathscr{E}^{*}$ is also nef and $\operatorname{deg}\left(\mathscr{E}^{*}\right)=$ $-\operatorname{deg}(\mathscr{E})+2(b-1) r \geq 0$. By Clifford's theorem, $p_{g}=h^{0}(\mathscr{E}) \leq \operatorname{deg}(\mathscr{E}) / 2+r \leq b r$. By (1.4), we have

$$
\operatorname{deg}\left(f_{*} \omega_{X}\right)=\operatorname{deg}(\mathscr{E})+\operatorname{deg}\left(\mathscr{E}^{\prime}\right) \geq 2(b-1) g
$$

If $\operatorname{deg}\left(f_{*} \omega_{X}\right)=2(b-1) g$, then we have $\operatorname{deg}\left(f_{*} \omega_{X / B}\right)=\operatorname{deg}\left(f_{*} \omega_{X}\right)-2(b-1) g=0$. Hence $f$ is locally trivial. The rest may be clear from Proposition 1.3. Q. E. D.

Lemma 1.5. If $b=0$, then $q(X) \leq r+1$.

Proof. Assume that $q \geq r+2$. Let $S$ be the minimal model of $X$ and let $\alpha: S \rightarrow$ $\operatorname{Alb}(S)$ be the Albanese map. Since $q>r+1$, it follows from [16, Theorem 2] that $\alpha(S)$ cannot be a surface. Hence $C=\alpha(S)$ is a non-singular irreducible curve of genus $q$. Let $\beta: X \rightarrow C$ be the fibration induced by $\alpha$. We denote by $h$ the genus of a general fibre $D_{1}$ of $\beta$. Since $b=0$, we have $\operatorname{deg}(\mathscr{E})=p_{g}-r$ and $\mu_{1}(\mathscr{E}) \geq p_{g} / r-1$. Hence $K_{X}-\left(p_{g} / r-1\right) D$ is pseudo-effective, and we have $\left(K_{X}-\left(p_{g} / r-1\right) D\right) D_{1} \geq 0$. Since $X$ is non-ruled, we have $D D_{1} \geq 2$. It follows that $2 h-2 \geq D D_{1}\left(p_{g} / r-1\right) \geq$ $2\left(p_{g} / r-1\right)$. On the other hand, we have $\operatorname{deg} \beta_{*} \omega_{X / C}=\chi\left(\mathcal{O}_{X}\right)-(h-1)(q-1) \geq 0$. Since $q \geq r+2$, we get

$$
\chi\left(\mathcal{O}_{X}\right) \geq(h-1)(q-1) \geq \frac{q-1}{r}\left(p_{g}-r\right) \geq \frac{r+1}{r}(\chi+1)
$$

which is impossible.

Q. E. D.

Now we can show the following :

Theorem 1.6. Let $f: X \rightarrow B$ be a relatively minimal fibration of genus $g \geq 2, b=$ $g(B)$, and assume that $X$ is of general type. Assume that the global sections of $f_{*} \omega_{X}$ generically generate a locally free subsheaf of rank $r$.

(1) If $b=0$, then $q(X) \leq \min \{g-r, r+1\}$.

(2) If $b=1$, then $q(X) \leq r$.

(3) If $b>1$ and $g>r$, then $q(X) \leq r$ and $g \leq r+r /(b-1)$.

(4) If $b>1$ and $g=r$, then $q(X) \leq b+g-1$ unless $X$ is a product of $B$ and $a$ curve of genus $g$. 
Proof. Assume that $b=0$. Then $\mathscr{F}=0$ and we have $q \leq g-r$ by Proposition 1.3. Hence we get (1) by Lemma 1.5.

Assume that $b>0$ and $g>r$. Then the inequality in Proposition 1.3 says that

$$
q \leq b+\operatorname{rk}(\mathscr{F})-(b-1)(g-r) \leq b+\operatorname{rk}(\mathscr{F})-(b-1) \leq \mathrm{rk}(\mathscr{F})+1 \leq r+1
$$

We assume that $q(X)=r+1$, and show that this eventually leads us to a contradiction. We have $\operatorname{rk}(\mathscr{F})=r, g=r+1$ (or $b=1$ ). Corollary 1.4 shows that $f$ is locally trivial and $\mathscr{E} \simeq \omega_{B}^{\oplus r}$. This cannot happen for $\mathrm{b}=1$, since $X$ is of general type. Hence we can assume that $b>1$. Since $f$ is locally trivial, we have an exact sequence

$$
0 \rightarrow f^{*} \omega_{B} \rightarrow \Omega_{X}^{1} \rightarrow \omega_{X / B} \rightarrow 0
$$

Then, as in $[12, \S 1]$, one can see that this sequence splits. Recall that $\mathscr{E} \otimes \omega_{B}^{-1} \simeq$ $\mathcal{O}_{B}^{\oplus r}$ is a subsheaf of $f_{*} \omega_{X / B}$. Hence, we have

$$
\begin{aligned}
h^{0}\left(\Omega_{X}^{1}\right) & =h^{0}\left(f^{*} \omega_{B}\right)+h^{0}\left(f_{*} \omega_{X / B}\right) \\
& \geq b+h^{0}\left(\mathscr{E} \otimes \omega_{B}^{-1}\right) \\
& =b+r
\end{aligned}
$$

which is impossible, since $r+1=q=h^{0}\left(\Omega_{X}^{1}\right)$ and $b>1$. Therefore, $q(X) \leq r$. By $q \geq$ $b$, we have $(b-1)(g-r) \leq \operatorname{rk}(\mathscr{F}) \leq r$. Hence $g \leq r+r /(b-1)$ when $b>1$ and $g>$ $r$.

Assume that $b>1$ and $g=r$. Then Proposition 1.3 gives us $q(X) \leq b+\operatorname{rk}(\mathscr{F})$ $\leq b+g$. If $q=b+g$, then $f$ is globally trivial as is well-known. $\quad$ Q. E. D.

We close the section with the following :

Lemma 1.7. Assume that $b=0, q=r+1$ and that the Albanese image is a curve $C$. Let $S$ be the minimal model of $X$. Then the Albanese pencil of $S$ is a locally trivial hyperelliptic fibration of genus $p_{g} / r, K_{S}^{2}=8 \chi\left(\mathcal{O}_{S}\right)$, and $\mathscr{E}$ is the direct sum of $r$ copies of $\mathcal{O}\left(p_{g} / r-1\right)$.

If $g=2 r+1$, then $X=S$ and $S$ is a double covering of $P=P^{1} \times C$ with branch locus $2\left(p_{g} / r+1\right)$ distinct fibers of $p_{1}: P \rightarrow P^{1}$. If $g>2 r+1$, then $m=2 r(g-2 r-1) /$ $\left(p_{g}+r\right)$ is an integer greater than 1 and $K_{X}^{2} \leq K_{S}^{2}-2 m$.

Proof. We use the same notation as in the proof of Lemma 1.5. Then the same argument there easily gives us $\mu_{1}(\mathscr{E})=p_{g} / r-1, D D_{1}=2, h=p_{g} / r$ and $\chi=(h-1)(q$ $-1)$. The last equality shows that $\alpha: S \rightarrow C$ is locally trivial. Since $D D_{1}=2, D_{1}$ is a double cover of $\boldsymbol{P}^{1}$. Hence it is a hyperelliptic curve. Since $\mu(\mathscr{E})=\mu_{1}(\mathscr{E})$ and $\boldsymbol{B}$ 
$=\mathbb{P}^{1}$, we see that $\mathscr{E} \simeq \mathcal{O}_{P^{1}}\left(p_{g} / r-1\right)^{\oplus r}$.

We have a holomorphic map $\phi: X \rightarrow \mathbb{P}=\mathbb{P}^{1} \times C$ putting $\phi=f \times \beta$. Since $D D_{1}=$ $2, \phi$ is of degree 2. Put $g=2 r+k$. It follows from Theorem 1.6 that $k$ is a positive integer. By the Riemann-Hurwitz formula, we see that the branch locus $B_{0}$ of $\phi$ is linearly equivalent to $2 \xi$, where $\xi=p_{1}^{*} \mathcal{O}_{P^{1}}(h+1)+p_{2}^{*}(\eta)$ and $\eta$ is a divisor of degree $k-1$ on $\mathbb{C}$. Furthermore, $X$ is birationally equivalent to a double covering $\boldsymbol{X}_{0}$ of $\boldsymbol{P}$ constructed in the total space of $[\xi]$ with branch locus $\boldsymbol{B}_{0}$. Note that $\boldsymbol{B}_{0}$ is free from multiple components. The dualizing sheaf of $X_{0}$ is induced by $K_{P}+\xi$. Hence $\chi\left(\mathcal{O}_{X_{0}}\right)=\chi+(k-1) h$ and $\omega_{X_{0}}^{2}=8 \chi+4(k-1)(h-1)$, where $\chi=\chi\left(\mathcal{O}_{S}\right)$.

If $k=1$, then $\chi\left(\mathcal{O}_{X_{0}}\right)=\chi$. Furthermore, $B_{0}$ consists of fibers of $p_{1}$ and $2 \eta=0$. In particular, since $B_{0}$ is smooth, $X_{0}$ is isomorphic to $X$. Note that $\eta \neq 0$, since, otherwise, $X$ is a product of $C$ and a curve of genus $h$ contradicting $q(X)=r+1=$ $g(\mathbb{C})$. Therefore, $\eta$ is a 2 -torsion element. Note further that $X=S$ in this case. Conversely, if we take a 2-torsion element $\eta \in \mathbb{P i c}^{0}(C)$ and construct a double covering $X_{0}$ of $P$ in $\left[p_{1}^{*} \mathcal{O}\left(p_{g} / r+1\right)+p_{2}^{*} \eta\right]$ with branch locus consisting of $2\left(p_{g} /\right.$ $r+1)$ distinct fibres of $p_{1}$, then an easy calculation shows that $X_{0}$ satisfies our requirements.

Assume that $k>1$. We take the canonical resolution $X^{*}$ of $X_{0}$ (see, [5]). Let $m_{l}$ denote the multiplicity of the singular point of $B_{0}$ appearing in the process of the canonical resolution. The difference of the invariants of $X_{0}$ and $X^{*}$ can be measured by the formula in $[5]$. Since $\chi\left(\mathcal{O}_{X} *\right)=\chi$, we have

$$
\sum_{l}\left[\frac{m_{i}}{2}\right]\left(\left[\frac{m_{l}}{2}\right]-1\right)=2(k-1) h
$$

Since $K_{S}^{2}=8 \chi$ and $K_{X}^{2} * \leq K_{X}^{2} \leq 8 \chi$, we have

$$
\sum_{i}\left(\left[\frac{m_{l}}{2}\right]-1\right)^{2} \geq 2(k-1)(h-1) .
$$

Since $k>1$, we can assume that $\left[m_{1} / 2\right]>1$. It follows from (1.5) and (1.6) that $2(k-1) \geq \Sigma\left(\left[m_{l} / 2\right]-1\right)$. Then, from $(1.5)$, we get $\Sigma\left(\left[\left[m_{l} / 2\right]-h\right)\left(\left[m_{l} / 2\right]-1\right)\right.$ $\geq 0$. This allows us to assume $\left[m_{1} / 2\right] \geq h$. Then the fibre $\Gamma_{1}$ of $p_{2}: P \rightarrow C$ passing through this singular point induces on $X^{*}$ a rational curve. Since $\alpha: S \rightarrow C$ is locally trivial, this implies $X^{*} \neq S$ and, hence, the equality does not hold in (1.6). Then, as above, we see that $\left[m_{1} / 2\right]=h+1$. Since every fibre of $\alpha$ is non-singular, the singular point must be a $2(h+1)$-ple point which becomes an ordinary $2(h+1)$-ple point after, say, $k_{1}$-times of blowing-ups $\left(k_{1} \geq 0\right)$, and $\Gamma_{1}$ is not a component of $B_{0}$. Hence, on $X^{*}$, the inverse image of $\Gamma_{1}$ consists of a non-singular curve of genus $h$, two $(-1)$-curves coming from the proper transform of $\Gamma_{1}$ and $2 k_{1}(-2)$-curves which are "infinitely near" $(-1)$-curves. These $(-1)$-curves must remain on $X$, since we have the holomorphic map $f: X \rightarrow \mathbb{P}^{1}$. Hence $K_{X}^{2} \leq K_{S}^{2}-2\left(k_{1}+1\right)=$ 
$8 \chi-2\left(k_{1}+1\right), m_{1}=\cdots=m_{k_{1}+1}=2 h+2$.

As in (1.5), (1.6), we get

$$
\sum_{i \geq k_{1}+2}\left[\frac{m_{i}}{2}\right]\left(\left[\frac{m_{i}}{2}\right]-1\right)=\left\{2(k-1)-\left(k_{1}+1\right)(h+1)\right\} h .
$$

and

$$
\sum_{i \geq k_{1}+2}\left(\left[\frac{m_{i}}{2}\right]-1\right)^{2} \geq\left\{2(k-1)-\left(k_{1}+1\right)(h+1)\right\}(h-1) .
$$

If $2(k-1)>\left(k_{1}+1\right)(h+1)$, then similarly as above, one can show that there is a singular point of $B_{0}$ of multiplicity $2(h+1)$ which becomes an ordinary $2(h+1)$-ple point after, say, $k_{2}$-times of blowing-ups. Let $\Gamma_{2}$ be the fibre of $p_{2}$ passing through this singular point. Then it creates two $(-1)$-curves and $2 k_{2}$ infinitely near $(-1)$ curves on $X$. Hence $K_{X}^{2} \leq 8 \chi-2\left(k_{1}+1\right)-2\left(k_{2}+1\right)$.

We can repeat such a procedure unless $2(k-1)$ is some multiple of $h+1$. Hence $m=2(k-1) /(h+1)$ is a positive integer and $K_{X}^{2} \leq K^{2}-2 m$. If $m=1$, one can easily see that the fibre of $p_{1}: P \rightarrow P^{1}$ passing through the singular point of multiplicity $2 h+2$ of $B_{0}$ is a multiple component of $B_{0}$, which is impossible.

Q. E. D.

\section{§ 2. Inequalities}

In this section, we give some inequalities generalizing one in $[14$, Lemma 3$]$ along an analogous line there. We freely use the notation in the previous section. In particular, let $0 \subset \mathscr{E}_{1} \subset \cdots \subset \mathscr{E}_{l}=\mathscr{E}$ be the Harder-Narashimhan filtration of $\mathscr{E}$. Put $d_{l}=M\left(\mathscr{E}_{l}\right) D$ and $a_{l}=2 g-2-d_{l}$ for $1 \leq i \leq l$. We put $d=d_{l}, a=a_{l}, M=M(\mathscr{E})$ and $Z=Z(\mathscr{E})$ for the sake of simplicity. If there are no danger of confusion, we also put $r_{t}=\operatorname{rk}\left(\mathscr{E}_{i}\right), \mu_{l}=\mu_{i}(\mathscr{E}), M_{l}=M\left(\mathscr{E}_{l}\right)$ and $Z_{l}=Z\left(\mathscr{E}_{l}\right)$.

Lemma 2.1. With the abcve notation, the following hold.

(1) $2 r_{t}-2 \leq d_{t} \leq 2 g-2$.

(2) Let $Z_{t}=\sum m_{j} G_{j}$ be the irreducible decomposition and put

$$
\alpha_{l}=\max _{J}\left\{m_{J} \mid D G_{j}>0\right\} .
$$

Then $t K_{X / B}+M_{l}-\mu_{l} D+Z_{l}$ is nef for any $t \geq \alpha_{l}$. In particular, $\left(a_{l}+1\right) K_{X}-\left(\mu_{l}+\right.$ $\left.2 a_{i}(b-1)\right) D$ is nef.

Proof. (1): We clearly have $d_{t} \leq 2 g-2$. Since $d_{l}$ equals to the degree of the linear system $\mid M_{l} \|_{D}$ which is of dimension $r_{t}-1$, Clifford's theorem shows $d_{i} \geq$ 
$2 r_{i}-2$.

(2) : Recall that $K_{X / B}$ and $M_{i}-\mu_{i} D$ are nef. Let $C$ be any irreducible curve on $X$. If $C$ is not a component of $Z_{i}$, then $Z_{i} C \geq 0$ and $\left(\alpha_{i} K_{X / B}+M_{i}-\mu_{i} D+Z_{i}\right) C \geq 0$. Assume that $C=G_{j}$ for some $j$. If $D G_{j}=0$, then $\left(\alpha_{i} K_{X / B}+M_{i}-\mu_{i} D+Z_{i}\right) G_{j}=\left(\alpha_{i}+\right.$ 1) $K_{X / B} G_{j} \geq 0$. If $D G_{j}>0$, then $\left(\alpha_{i} K_{X / B}+M_{i}-\mu_{i} D+Z_{i}\right) G_{j}=\left(\alpha_{i}-m_{j}\right) K_{X / B} G_{j}+$ $m_{j}\left(K_{X / B}+G_{j}\right) G_{j}+\left(Z_{i}-m_{j} G_{j}\right) G_{j} \geq 0$. Hence $\alpha_{i} K_{X / B}+M_{i}-\mu_{i} D+Z_{i}=\left(\alpha_{i}+1\right) K_{X}-$ $\left(\mu_{i}+2 \alpha_{i}(b-1)\right) D$ is nef. Since $a_{i}=D Z_{i}$, we always have $a_{i} \geq \alpha_{i}$. Therefore, $\left(a_{i}+1\right)$ $K_{X}-\left(\mu_{i}+2 a_{i}(b-1)\right) D$ is nef.

Q. E. D.

Lemma 2.2. If $\mathrm{rk}(\mathscr{F}) \leq r-1$, then $\operatorname{deg}(\mathscr{E}) \geq p_{g}-r+b(r-\operatorname{rk}(\mathscr{F}))$. If $\operatorname{rk}(\mathscr{F})$ $=r$, then $\operatorname{deg}(\mathscr{E}) \geq 2\left(p_{g}-r\right)$.

Proof. By the Riemann-Roch theorem and $p_{g}=h^{0}(\mathscr{E})$, we get $\operatorname{deg}(\mathscr{E})=p_{g}+$ $r(b-1)-h^{1}(\mathscr{E})$. Since $\mathscr{F}$ is nef, we have $\operatorname{deg}(\mathscr{F}) \geq 0$. Hence, by (1.2), we have $h^{1}(\mathscr{E}) \leq b \mathrm{rk}(\mathscr{F})$. If $\mathrm{rk}(\mathscr{F})=r$, then $\mathscr{F}=\mathscr{E}$ and Clifford's theorem shows $p_{g}=$ $h^{0}(\mathscr{E}) \leq \operatorname{deg}(\mathscr{E}) / 2+r$. Hence $\operatorname{deg}(\mathscr{E}) \geq 2\left(p_{g}-r\right)$.

Q. E.D.

Corollary 2.3. If $r>1$ and $p_{g} \geq \min \{(3 r-2) b+r+1,2(g-1) b+g+q+1\}$, then the canonical map of $X$ separates fibers of $f$.

Proof. Let $L$ be a line bundle of degree $2 b+1$ on $B$. Then it is very ample.

Assume that $p_{g} \geq(3 r-2) b+r+1$. Since $p_{g}>b r$, we have $\operatorname{rk}(\mathscr{F})<r$ by Corollary 1.4, and Lemma 2.2 shows that $\operatorname{deg}(\mathscr{E}) \geq p_{g}-r+b$. We have $\operatorname{deg}(\mathscr{E}(-L))$ $\geq p_{g}-r+b-r(2 b+1) \geq(\mathrm{r}-1)(b-1)$ by assumption. Hence, by [8, Corollary], $L$ can be chosen so that $H^{0}(\mathscr{E}(-L)) \neq 0$. Since $\left|f^{*} L\right|+\left(K_{X}-f^{*} L\right)$ is a subsystem of $\left|K_{X}\right|$, the canonical map separates fibers of $f$.

Assume that $p_{g} \geq 2(g-1) b+g+q+1$. Since $\operatorname{deg}\left(f_{*} \omega_{X}\right)=\chi\left(\mathcal{O}_{X}\right)+(g+1)(b$ $-1)$, we have $\operatorname{deg}\left(f_{*} \omega_{X}(-L)\right) \geq(g-1)(b-1)$. Hence, as above, the canonical map can separate fibers also in this case.

Q. E. D.

\section{Lemma 2.4.}

$$
K_{X}^{2} \geq \frac{4 g(g-1)-d_{1}^{2}}{2 g-d_{1}-1} \mu_{1}(\mathscr{E})+\frac{2\left(2 g-2-d_{1}\right)^{2}}{2 g-d_{1}-1}(b-1)
$$

In particular,

$$
K_{X}^{2} \geq \frac{4 g(g-1)-d_{1}^{2}}{2 g-d_{1}-1} \mu(\mathscr{E})+\frac{2\left(2 g-2-d_{1}\right)^{2}}{2 g-d_{1}-1}(b-1)
$$

Proof. For each $i$, we have 


$$
\begin{aligned}
K_{X}^{2} & =K_{X}\left(M_{i}+Z_{i}\right) \\
& =\left(K_{X}-\mu_{1} D\right)\left(M_{i}-\mu_{i} D\right)+\left(2 g-2-a_{i}\right) \mu_{1}+2(g-1) \mu_{i}+K_{X} Z_{i} \\
& \geq\left(2 g-2-a_{i}\right) \mu_{1}+2(g-1) \mu_{i}+K_{X} Z_{i}
\end{aligned}
$$

Since $\left(\left(a_{i}+1\right) K_{X}-\left(\mu_{i}+2 a(b-1)\right) D\right) Z_{i} \geq 0$, we have

$$
K_{X} Z_{i} \geq \frac{a_{i}}{a_{i}+1}\left(\mu_{i}+2 a_{i}(b-1)\right) .
$$

Now, put $i=1$. It follows from (2.1) and (2.2) that

$$
K_{X}^{2} \geq 4(g-1) \mu_{1}-\frac{a_{1}^{2}}{a_{1}+1}\left(\mu_{1}-2(b-1)\right)
$$

Hence we get the inequalities, if we note $\mu_{1} \geq \mu(\mathscr{E})$.

Q. E. D.

Corollary 2.5. If $\operatorname{deg}(\mathscr{E}) \geq 2 r(b-1)$, then

$$
K_{X}^{2} \geq \frac{4 g(g-1)}{2 g-1}\left(\mu(\mathscr{E})+2\left(1-\frac{1}{g}\right)(b-1)\right) .
$$

Proof. Since $a_{1}=2 g-2-d_{1} \leq 2 g-2 r_{1} \leq 2 g-2$, we have $a_{1}^{2} /\left(a_{1}+1\right) \leq 4(g$ $-1)^{2} /(2 g-1)$. Since $\mu_{1} \geq \mu(\mathscr{E}) \geq 2(b-1)$, (2.3) gives the inequality. Q. E. D.

When $d$ is small enough, we can give a better bound.

Lemma 2.6. Assume that $0<d \leq \min \{2 g-r, 2 g-3\}$ and $\operatorname{deg}(\mathscr{E}) \geq 2(b-1) d /$ $(2 g-1)$. Then

$$
K_{X}^{2} \geq \frac{4 g(g-1)}{(2 g-1) r-d}\left(\operatorname{deg}(\mathscr{E})+2(b-1)\left(r-\frac{d+r}{g}\right)\right)
$$

Proof. $(a+1) K_{X}-\left(\mu_{l}+2 a(b-1)\right) D$ is nef by Lemma 2.1. Since $K_{X}-\mu_{1} D$ is pseudo-effective, we have $\left(K_{X}-\mu_{1} D\right)\left((a+1) K_{X}-\left(\mu_{l}+2 a(b-1)\right) D\right) \geq 0$. If follows from this and (1.1) that

$$
\begin{aligned}
(a+1) K_{X}^{2} & \geq 2(g-1)\left((a+1) \mu_{1}+\mu_{l}+2 a(b-1)\right) \\
& \geq 2(g-1)\left((a-r+2) \mu_{1}+\operatorname{deg}(\mathscr{E})+2 a(b-1)\right) .
\end{aligned}
$$

On the other hand, (2.1) and (2.2) for $i=l$ give us 


$$
\begin{aligned}
K_{X}^{2} & \geq(2 g-2-a) \mu_{1}+2(g-1) \mu_{l}+K_{X} Z \\
& \geq(2 g-2-a) \mu_{1}+2(g-1) \mu_{l}+\frac{a}{a+1}\left(\mu_{l}+2 a(b-1)\right) .
\end{aligned}
$$

Hence it follows from (1.1) that

$$
\begin{aligned}
(a+1) K_{X}^{2} \geq & -(a((r-1)(2 g-1)+a+1)-2(g-1)(a-r+2)) \mu_{1} \\
& +((2 g-1) a+2 g-2) \operatorname{deg}(\mathscr{E})+2 a^{2}(b-1) .
\end{aligned}
$$

Note that we have $2(g-1)(a-r+2) \leq((r-1)(2 g-1)+a+1) a$.

Since $a>0$, the desired inequality follows from $(2.4)$ when $((r-1)(2 g-1)+$ $a+1)) \mu_{1} \geq(2 g-1) \operatorname{deg}(\mathscr{E})-2(b-1)(2 g-2-a)$ and, otherwise, it follows from (2.5).

Q.E.D.

By using the same method, one can also get a slight improvement of $[15$, Corollary 3$]$.

Lemma 2.7. Let $f: X \rightarrow B$ be a relatively minimal fibration of genus $g \geq 2, b=$ $g(B)$, and put $h=q(X)-b$. If $g-h>0$, then

$$
K_{X / B}^{2} \geq \frac{4 g(g-1)}{(2 g-1)(g-h)} \operatorname{deg}\left(f_{*} \omega_{X / B}\right)
$$

When $f$ is not locally trivial, the equality holds only if $g-h=1$.

Proof. By [3, Theorem 3.1], $f_{*} \omega_{X / B}=\mathscr{H} \oplus \mathcal{O}_{B}^{\oplus h}$. Hence $\operatorname{deg}(\mathscr{H})=\operatorname{deg} f_{*} \omega_{X / B}$ and $\operatorname{rk}(\mathscr{H})=g-h$. Since $\mathscr{H}$ is a direct factor of $f_{*} \omega_{X / B}$, it is nef.

Let $0 \subset \mathscr{H}_{1} \subset \cdots \subset \mathscr{H}_{k}=\mathscr{H}$ be the Harder-Narashimhan filtration for $\mathscr{H}$. The natural sheaf homomorphism $f^{*} \mathscr{H}_{1} \rightarrow f^{*} f_{*} \omega_{X / B} \rightarrow \omega_{X / B}$ induces a rational map $\phi: X$ $\rightarrow \mathbb{P}\left(\mathscr{H}_{1}\right)$. Let $M$ be the pull-back of a tautological divisor by $\phi$. Then $K_{X / B} \equiv M+$ $Z$ with an effective divisor $Z$, and $M-\mu_{1}(\mathscr{H}) D$ is nef, where $D$ denotes a general fibre of $f$. Put $a=D \mathbb{Z}$. Since $\mu_{1}(\mathscr{H}) \geq \mu(\mathscr{H})=\operatorname{deg} f_{*} \omega_{X / B} /(g-h)$, it is sufficient to show

$$
K_{X / B}^{2} \geq \frac{4 g(g-1)}{2 g-1} \mu_{1}(\mathscr{H}) .
$$

Similarly as in Lemma 2.1 , one can show that $(a+1) K_{X / B}-\mu_{1}(\mathscr{H}) D$ is nef. Hence $K_{X / B} Z \geq a \mu_{1}(\mathscr{H}) /(a+1)$ and we get

$$
K_{X / B}^{2} \geq((a+1)(4 g-4-a)+a) \mu_{1}(\mathscr{H}) /(a+1)
$$


similarly as in (2.3). Since $a \leq 2 g-2$, we get (2.6) with equality holding only if $a$ $=2 g-2\left(\right.$ hence $\operatorname{rk}\left(\mathscr{H}_{1}\right)=1$ since $2 g-2-a \geq 2 \operatorname{rk}\left(\mathscr{H}_{1}\right)-2$ by Clifford's theorem).

Q. E. D.

Proposition 2.8. If $f: X \rightarrow B$ is a relatively minimal fibration of genus $g \geq 2$ which is not locally trivial. Then

$$
q(X)-b \leq \frac{g(5 g-2)}{3(2 g-1)}<\frac{5 g+1}{6}
$$

When $f$ is of hyperelliptic type,

$$
q(X)-b \leq \begin{cases}\frac{\left(5 g^{2}+g-1\right) g}{(2 g-1)(3 g+1)}, & \text { if } g \text { is even, } \\ \frac{\left(5 g^{3}-6 g^{2}+5 g-1\right) g}{(2 g-1)\left(3 g^{2}-2 g+2\right)}, & \text { if } g \text { is odd. }\end{cases}
$$

Proof. If $f$ is not locally trivial, its slope $\lambda(f)=K_{X / B}^{2} / \operatorname{deg}\left(f_{*} \omega_{X / B}\right)$ is well-defined and satisfies $\lambda(f) \leq 12$ by $[15$, Theorem 2]. If $f$ is a hyperelliptic fibration, then $[7$, Theorem 4.0 .4$]$ shows

$$
\lambda(f) \leq \begin{cases}\frac{4(g-1)(3 g+1)}{g^{2}}, & \text { if } g \text { is even, } \\ \frac{4\left(3 g^{2}-2 g+2\right)}{g^{2}+1}, & \text { if } g \text { is odd. }\end{cases}
$$

Since we have $\lambda(f) \geq 4 g(g-1) /(2 g-1)(g-h)$ by Lemma 2.7, an easy calculation shows the assertions.

Q. E. D.

Corollary 2.9. Let the situation be as in Theorem 1.6, and assume that $b>0, g$ $=r \geq 2$. If $q(X)=b+g-1$, then one of the following holds :

(1) $p_{g}=g b-1, g \leq 3, f$ is locally trivial and $K_{X}^{2}=8 \chi\left(\mathcal{O}_{X}\right)$.

(2) $p_{g} \geq g b, g \leq 6$, and

$$
K_{X}^{2} \geq \begin{cases}\frac{4(g-1)}{2 g-1}\left(g \chi\left(\mathcal{O}_{X}\right)-\left(g^{2}-5 g+2\right)(b-1)\right) & \text { if } g \geq 3, \\ 4 p_{g}-4 & \text { if } g=2 .\end{cases}
$$

Proof. We have $\operatorname{deg}\left(f_{*} \omega_{X / B}\right)=p_{g}-g b+1$. Since it is a non-negative integer, we get $p_{g} \geq g b-1$.

Assume that $p_{g}=g b-1$. Then $f$ is locally trivial, and we get $q-b \leq(g+1) / 2$ 
by the proof of $[15$, Corollary 3]. Since $q-b=g-1$, we get $g \leq 3$.

Assume that $p_{g} \geq g b$. Since $f$ is not locally trivial and $q-b=g-1$, it follows from Lemma 2.7 and Proposition 2.8 that $K_{X / B}^{2} \geq(4 g(g-1) /(2 g-1)) \operatorname{deg} f_{*} \omega_{X / B}$ and $g \leq 6$, respectively. We also have $K_{X / B}^{2} \geq 4 \operatorname{deg} f_{*} \omega_{X / B}$ by $[15$, Theorem 1]. Hence we get (2).

Q.E.D.

\section{§ 3. Surfaces whose Canonical Map Is a Pencil}

From now on, we let $S$ be a minimal surface of general type with $p_{g} \geq 2$. In this section, we assume that the canonical image is a curve $\Sigma$. Let $\sigma: X \rightarrow S$ be the elimination of the base points of the variable part of $|K|$. Then taking the Stein factorization, we get a relatively minimal fibration $f: X \rightarrow B$ of genus $g, b=g(B)$. In this case, $\mathscr{E}$ is a line bundle and $M(\mathscr{E}) \equiv \operatorname{deg}(\mathscr{E}) D$. Hence $d=M(\mathscr{E}) D=0$.

Theorem 3.1. Assume that the canonical map of $S$ is composed of a pencil. Then $b=q=1$ or $b=0, q \leq 2$. If $q=2$, then $g \geq 3$. Furthermore,

$$
K^{2} \geq K_{X}^{2} \geq \frac{4 g(g-1)}{2 g-1}\left(p_{g}+(b-1)\left(3-\frac{2}{g}\right)\right)
$$

Proof. The statement for $b, q$ follows from Theorem 1.6. Then, since $b \leq 1$ and since $\mathscr{E}$ is a line bundle with $h^{0}(\mathscr{E})=p_{g}>1$, we have $\operatorname{deg}(\mathscr{E})=p_{g}-1+b$. Hence we get (3.1) by Lemma 2.4 putting $d=d_{1}=0, r=1$.

Q. E. D.

Remark 3.2. The statement for $b, q$ in Theorem 3.1 already can be found in [12]. Unfortunately, (3.1) may not be sharp : When $g=2$ and $p_{g} \geq 3$, we can find the following bound in [13]:

$$
K^{2} \geq \begin{cases}4 p_{g}-6, & \text { if }(b, q)=(0,0) \\ 4 p_{g}-4, & \text { if }(b, q)=(0,1) \\ 4 p_{g}, & \text { if }(b, q)=(1,1)\end{cases}
$$

When $b=0$, we can write $|K|=\left|\left(p_{g}-1\right) D_{0}\right|+Z_{0}$, where $D_{0}=\sigma_{*} D$ and $Z_{0}=$ $\sigma_{*} Z$.

Lemma 3.3. Let the notation be as above and assume that $b=0$.

(1) If $q=1$, then $K^{2} \geq 4 p_{g}-4$ with equality holding only if the Albanese pencil is hyperelliptic.

(2) If $D_{0}^{2}=0$, then $K^{2} \geq 2(g-1)\left(p_{g}-1\right)$.

(3) If $D_{0}^{2}>0$, then $K^{2} \geq \max \left\{D_{0}^{2}\left(p_{g}-1\right)^{2},\left(2 g-2-D_{0}^{2}\right)\left(p_{g}-1\right)\right\}$. In particu- 
lar, $K^{2} \geq 2(g-1)\left(1-1 / p_{g}\right)\left(p_{g}-1\right)$.

Proof. (1) : Let $\alpha: S \rightarrow \operatorname{Alb}(S)$ be the Albanese map, and let $D_{1}$ be a general fibre of $\alpha$. Since $K-\left(p_{g}-1\right) D_{0}$ is pseudo-effective, we have $0 \leq\left(K-\left(p_{g}-1\right) D_{0}\right) D_{1}$ $=2 h-2-D_{0} D_{1}\left(p_{g}-1\right) \leq 2 h-2-2\left(p_{g}-1\right)$, where $h=g\left(D_{1}\right)$. Hence $h \geq p_{g}$ with equality holding only if $D_{1}$ is a hyperelliptic curve. On the other hand, we have $K^{2} \geq(4-4 / h) \chi$ by $[15$, Theorem 2$]$. Hence $K^{2} \geq\left(4-4 / p_{g}\right) p_{g}=4 p_{g}-4$.

(2) : Since $K$ is nef, we have $K^{2}=\left(p_{g}-1\right) K D_{0}+K Z_{0}=2(g-1)\left(p_{g}-1\right)+K Z_{0}$ $\geq 2(g-1)\left(p_{g}-1\right)$.

(3): We have $Z=\sigma^{*} Z_{0}+\sum\left(\left(p_{g}-1\right) m_{i}+1\right) E_{i}$, where $m_{i}$ denotes the multiplicity of a base point of $\left|D_{0}\right|$ appearing in $\sigma$, and $E_{i}$ is the inverse image of the base point. Hence $2 g-2-\sum m_{i}=K D_{0}=\left(p_{g}-1\right) D_{0}^{2}+D_{0} Z_{0} . \quad K^{2}=(2 g-2-$ $\left.\sum m_{i}\right)\left(p_{g}-1\right)+K Z_{0}=\left(p_{g}-1\right)^{2} D_{0}^{2}+\left(K+\left(p_{g}-1\right) D_{0}\right) Z_{0} \geq\left(p_{g}-1\right)^{2} D_{0}^{2}$. We also note that $D_{0}^{2} \geq \sum m_{i}$. Hence $K^{2} \geq\left(2 g-2-D_{0}^{2}\right)\left(p_{g}-1\right)$.

Q. E. D.

Corollary 3.4. Let $S$ be a minimal surface of general type whose canonical map is composed of a pencil. Then $K^{2} \geq 4 p_{g}-7$.

Proof. By Remark 3.2, we can assume that $g \geq 3$. By Lemma 3.3, we only have to consider the case that $b=0, D_{0}^{2}>0$ and $p_{g} \leq 4$. If $p_{g}=4$, then Lemma 3.3, (3) implies that $K^{2} \geq 3\left(p_{g}-1\right)=4 p_{g}-7$. Assume that $p_{g}=3$. If $D_{0}^{2} \geq 2$, then we are done. If $D_{0}^{2}=1$, then $K D_{0}=2+D_{0} Z_{0}$. Since $K D_{0}+D_{0}^{2}$ is even, $D_{0} Z_{0}$ is a positive odd integer. It follows $K^{2} \geq(3-1)^{2}+(3-1)=6=4 p_{g}-6$. Assume that $p_{g}=2$. Then $K^{2} \geq 1=4 p_{g}-7$.

Q. E. D.

Corollary 3.5. Let the notation and assumption be as above. Assume that the variable part of $|K|$ is free from base points, when $b=0$. Then the following hold.

(1) If $b=q=1$, then $g \leq 5$.

(2) If $b=0$ and $p_{g} \geq 20-9 q$, then $g \leq 5$.

Proof. By Miyaoka-Yau's inequality, we have $K^{2} \leq 9 \chi$. Hence (1) and (2) follow from (3.1) and Lemma 3.3.

Q. E. D.

When $q=2$, we can say more :

Theorem 3.6. Let $S$ be a minimal surface of general type with $q=2$ whose canonical map is composed of a pencil of genus $g$. Assume that the Albanese map is not surjective. Then $K^{2}=8 \chi$ and the Albanese pencil is a locally trivial hyperelliptic fibration of genus $p_{g}$. Furthermore, $g=3$ and $S$ is an example of Beauville $[1,2.5]$ except possibly when $\left(p_{g}, g\right)=(2,6),(2,9)$ or $(3,7)$.

Proof. Except for the last sentence, this is clear from Lemma 1.7. Assume that 
$g>3$ and put $m=2(g-3) /\left(p_{g}+1\right)$. Then $D_{0}^{2} \geq 2 m$ as we saw in the proof of Lemma 1.7. Since $K^{2}=8 \chi=8\left(p_{g}-1\right)$, Lemma 3.3 gives us $8 \geq D_{0}^{2}\left(p_{g}-1\right) \geq 2 m\left(p_{g}\right.$ $-1)$. Since $m \geq 2$, we have $2\left(p_{g}+1\right) \geq(g-3)\left(p_{g}-1\right) \geq\left(p_{g}+1\right)\left(p_{g}-1\right)$. Since $m$ is an integer, we obtain the list of the exceptions.

Q.E.D.

\section{§4. Surfaces with High Camonical Degree}

In this section, we assume that the canonical map of $S$ induces a rational map $\phi_{K}: S \rightarrow \Sigma \subset \boldsymbol{P}^{p_{g}-1}$ of degree $d_{c a n}>1$ onto the image $\Sigma$.

The following lemma due to Xiao [14, Lemma 1] guarantees that $\Sigma$ is ruled by rational curves of small degree when $d_{c a n}$ is large. See also [10].

Lemma 4.1. If there exists a positive integer $\delta$ such that

$$
\operatorname{deg} \Sigma<\frac{2(\delta+1)}{\delta+2}\left(p_{g}-1-\frac{9}{8}(\delta+1)\right)
$$

then $\Sigma$ has a pencil of rational curves of degree $\leq \delta$. Furthermore, when $\delta=1$, the above inequality can be weakened to

$$
\operatorname{deg} \Sigma<\frac{4}{3}\left(p_{g}-3\right)
$$

except if $p_{g}=10$ and $(\Sigma, \mathcal{O}(1)) \simeq\left(\mathbb{P}^{2}, \mathcal{O}(3)\right)$.

Assume that $\Sigma$ is ruled by rational curves of degree $\delta$. Let $\Lambda$ be a pencil of curves on $S$ induced by the ruling of $\Sigma$ via $\phi_{K}$. Let $\sigma: X \rightarrow S$ be the composite of blowing-ups which eliminates $\operatorname{Bs} \Lambda$. Then, taking the Stein factorization if necessary, we get a relatively minimal fibration $f: X \rightarrow B$. As before, we denote by $g$ the genus of a general fibre $D$ of $f$ and put $b=g(B)$.

Let $\mathscr{E}$ be the locally free subsheaf of $f_{*} \omega_{X}$ generically generated by its global sections. Since $\mathbb{D}$ is mapped onto a rational curves of degree $\delta$, the restriction map $H^{0}\left(K_{X}\right) \rightarrow H^{0}\left(K_{D}\right)$ is of rank $\leq \delta+1$. Hence $r=\operatorname{rk}(\mathscr{E}) \leq \delta+1$. Put $d=M(\mathscr{E}) \mathbb{D}$ as before. Let $\phi: X \rightarrow \mathbb{P}(\mathscr{E})$ be, as in 1.2, the rational map associated with $f^{*} \mathscr{E} \rightarrow \omega_{X}$. Then, by the choice of $\mathscr{E}$, the canonical map $\Phi_{K_{X}}$ is a composite of $\phi$ and the rational map of $\boldsymbol{P}(\mathscr{E})$ induced by $H(\mathscr{E})$ which we denote by $\Phi_{H}$.

Lemma 4.2. Assume that the canonical image is ruled by rational curves of degree $\delta$.

(1) $d_{\text {can }}$ is a multiple of $d / \delta$. If $\Phi_{H}$ separates fibers of $\mathbb{P}(\mathscr{E}) \rightarrow \mathbb{B}$, then $d=d_{\text {can }} \delta$. If $d_{c a n}$ is a prime number, then $d=d_{c a n} \delta$. 
(2) If $g=r$, then $f$ is of hyperelliptic type, $d=2 \delta$ and $d_{c a n}$ is even.

Proof. (1) : Since the image of $D$ under the canonical map is a rational curve of degree $\delta, d$ is a multiple of $\delta$, and $d / \delta$ equals the degree of $\left.\Phi_{K_{X}}\right|_{D}$, hence, $\phi$ is of degree $d / \delta$ onto its image.

(2) : Since $\operatorname{rk}(\mathscr{E})=g$, the restriction map $H^{0}\left(K_{X}\right) \rightarrow H^{0}\left(K_{D}\right)$ is surjective. By the assumption, it follows that $D$ is mapped onto a rational curve via its canonical map. Hence $D$ is a hyperelliptic curve. By what we saw above, $\phi$ is of degree 2 onto the image. Hence $d_{c a n}$ must be even.

Q. E. D.

Note that $S$ has no pencil of hyperelliptic curves if $d_{c a n}$ is odd. Hence Theorem 1.6, Lemma 1.7 and Lemma 4.2 give us the following generalization of [16, Theorem 3].

Theorem 4.3. Assume that $\sum$ is ruled by rational curves of degree $\delta$. Assume further that $g>\delta+1$ or $d_{c a n}$ is odd. Then $q \leq \delta+2$. If $q=\delta+2$, then $b=0$ and $g \geq$ $2 \delta+3$. If $d_{c a n}$ is odd and $q=\delta+2$, the Albanese image of $S$ is a surface.

Lemma 4.4. Suppose that $b>1$ and $g=\delta+1$.

(1) Assume that $\delta=1$. Then $d_{c a n}$ is an even integer not exceeding 10. If $d_{\text {can }}$ $=10$, then $b=q=2, p_{g}=3$. If $d_{c a n}=8$, then $\left(b, q, p_{g}\right)=(2,2,3),(2,3,3)$ or $(3,3$, 4). If $d_{c a n}=6$, then $\left(b, q, p_{g}\right)=(2,2,3),(2,2,4),(2,3,3),(3,3,4),(3,3,5)$ or $(4,4,6)$.

(2) If $\delta=2$ and $d_{c a n}=6$, then $\left(b, q, p_{g}\right)=(2,2,4),(2,2,6),(3,3,6)$ or (4, $4,9)$.

Proof. We can assume that $\mathscr{E}=f_{*} \omega_{X}$. Put $H=H\left(f_{*} \omega_{X}\right)$. Since $\operatorname{deg} f_{*} \omega_{X / B} \geq$ 0 , we have

$$
p_{g} \geq q+\delta(b-1)-1
$$

(1) : Though this is essentially contained in $[13$, p. 74$]$, we give a proof for the sake of completeness. Put $d_{c a n}=2 m$. Then $\Phi_{H}$ is a map of degree $m$ onto the image $\Sigma$. Hence $H^{2} \geq m \operatorname{deg} \Sigma$. Since $H^{2}=\operatorname{deg} f_{*} \omega_{X}=\chi+3(b-1)$ and $\operatorname{deg} \Sigma \geq p_{g}-2$, we get

$$
(m-1) p_{g} \leq 3 b-q+2 m-2 .
$$

From (4.1) and (4.2), we get $m q+(m-3) b \leq 4 m-4$. If $q \geq 3$, then we have $m \leq$ 4 , since $b \geq 2$. Assume that $q=b=2$. Since $p_{g} \geq 3$, it follows from (4.2) that $4=$ $3 b-q \geq m-1$. Hence we get $m \leq 5$. The rest follow from an easy calculation.

(2) : Let $V$ be the image of $\phi: X \rightarrow \mathbb{P}\left(f_{*} \omega_{X}\right)$. Then $V$ is numerically equivalent to $2 H-v F$ with an integer $v$. Since $V$ is a relative hyperquadric of rank 3 , one can 
easily show $3 v \leq 2 \operatorname{deg}\left(f_{*} \omega_{X}\right)$ (see, e. g., [6]). Since $H$ induces a map of degree 3 , we have $H^{2}(2 H-v F) \geq 3 \operatorname{deg} \Sigma$, that is, $2 \operatorname{deg}\left(f_{*} \omega_{X}\right)-v \geq 3 \operatorname{deg} \Sigma$. Hence $\operatorname{deg}\left(f_{*} \omega_{X}\right)$ $\geq(9 / 4) \operatorname{deg} \Sigma$. On the other hand, since $\Sigma$ is not ruled by straight lines, Lemma 4.1 gives us $\operatorname{deg} \Sigma \geq(4 / 3)\left(p_{g}-1-9 / 4\right)$. Therefore, $\operatorname{deg}\left(f_{*} \omega_{X}\right) \geq 3 p_{g}-9$. Since $\operatorname{deg}\left(f_{*} \omega_{X}\right)=\chi+4(b-1)$, we have

$$
2 p_{g} \leq 4 b-q+6 \text {. }
$$

It follows from (4.1) and (4.3) that $q \leq 4$. Furthermore, since $p_{g} \geq 4$, we get

$$
\begin{aligned}
& (b, q)=(2,2): 4 \leq p_{g} \leq 6 \\
& (b, q)=(2,3): p_{g}=4,5 \\
& (b, q)=(2,4): p_{g}=5 \\
& (b, q)=(3,3): p_{g}=6,7 \\
& (b, q)=(3,4): p_{g}=7 \\
& (b, q)=(4,4): p_{g}=9
\end{aligned}
$$

It is known that surfaces with degree $p_{g}-2$ in $\boldsymbol{P}^{p_{g}}{ }^{-1}$ is ruled by straight lines unless it is the Veronese surface, $p_{g}=6$. Hence, if $p_{g} \neq 6$, we can assume that $\operatorname{deg} \Sigma \geq p_{g}-1$. Since $\operatorname{deg} f_{*} \omega_{X} \geq(9 / 4)\left(p_{g}-1\right)$, we have

$$
\operatorname{deg} f_{*} \omega_{X} \geq \begin{cases}7, & \text { if } p_{g}=4, \\ 9, & \text { if } p_{g}=5, \\ 14, & \text { if } p_{g}=7 .\end{cases}
$$

Hence we can exclude several cases and get (2).

Q. E. D.

In $\left[14\right.$, Theorem 5], it is shown that there is a bound of $q, g$ when $d_{c a n} \geq 5$. Now we can give a bound on $q$.

Theorem 4.5. Let $S$ be a surface of general type whose canonical map is a rational map of degree $d_{c a n}>4$ onto its image.

(1) If $d_{c a n} \geq 7$, then $q \leq 3$ except possibly when $d_{c a n}=7, p_{g}=10, q=4, K^{2}=63$ and $\Sigma$ is $\boldsymbol{P}^{2}$ embedded into $\boldsymbol{P}^{9}$ by $|\mathcal{O}(3)|$.

(2) If $d_{\text {can }}=6$, then $q \leq 5$.

(3) If $d_{c a n}=5$, then $q \leq 12$, and $q \neq 12$ when $p_{g}>136$.

Proof. (1) : Assume that $q \geq 4$. Miyaoka-Yau's inequality gives us

$$
\operatorname{deg} \Sigma \leq K^{2} / d_{c a n} \leq 9 \chi / d_{c a n} \leq\left(9 / d_{c a n}\right)\left(p_{g}-3\right)
$$

Hence Lemma 4.1 implies that $\Sigma$ is ruled by lines unless we are in the case excepted 
in (1). But then, Theorem 4.3 and Lemma 4.4 give us $q \leq 3$, a contradiction.

(2) : Assume that $q \geq 6$. By the same reasoning as above, Lemma 4.1 implies that $\Sigma$ is ruled by rational curves of degree $\delta \leq 2$. In this case, however, Theorem 4.3 and Lemma 4.4 give us $q \leq 4$, a contradiction.

(3) : Assume that $q \geq 13$. By the same reasoning as above, Lemma 4.1 implies that $\Sigma$ is ruled by rational curves of degree $\delta \leq 8$. But, Theorem 4.3 shows $q \leq 10$ contradicting our initial assumption. Quite similarly, assuming $q=12$ and $p_{g}>136$, we can show that $\Sigma$ is ruled by rational curves of degree $\delta \leq 9$. But Theorem 4.3 tells us $q \leq 11$.

Q. E. D.

Remark 4.6. In the above theorem, (1) and (2) respectively can weaken the assumption on $p_{g}$ in $[16$, p. 602, Corollary] and [11, Theorem 3$]$.

As for $g$, we can show, for example, the following :

Proposition 4.7. Let the notation and assumption be as above.

(1) If $d_{c a n}=6$ and $p_{g}>190$, then $g \leq 16$.

(2) If $d_{c a n}=5$ and $p_{g}>1324$, then $g \leq 44$.

Proof. We show only (2), because (1) can be treated similarly if we note that $d=6 \delta$ holds when $p_{g}$ is large enough by Corollary 2.3 and Lemma 4.2.

If $p_{g}>1324$, then

$$
\operatorname{deg} \Sigma \leq \frac{9}{5}\left(p_{g}+1\right)<\frac{2(9+1)}{9+2}\left(p_{g}-1-\frac{9}{8}(9+1)\right) .
$$

Hence, by Lemma 4.1, $\Sigma$ is ruled by rational curves of degree $\delta \leq 9$. We assume $g$ $\geq 45$ and show that this leads us to a contradiction. By Theorem 1.6, we can suppose $b \leq 1$. By Lemma 4.2, we have $d=5 \delta$. Since $5 \delta \leq 45<2 g-10 \leq 2 g-\delta-1$, it follows from Lemma 2.6 (and Lemma 2.4 when $\mathscr{E}$ is semi-stable) that $K^{2} \geq$ $(1584 / 169)\left(p_{g}-28\right)$. However, since $p_{g}>728$, this contradicts Miyaoka-Yau's inequality $K^{2} \leq 9\left(p_{g}+1\right)$. Hence $g \leq 44$.

Q.E. D.

\section{References}

[1] Beauville, A., L'application canonique pour les surfaces de type général, Invent. Math., 55 (1979), 121-140.

[2] L'inegalite $p_{g} \geq 2 q-4$ pour les surfaces de type général, Bull. Soc. Math. France, 110 (1982), 343-346.

[ 3 ] Fujita, T., On Kähler fiber spaces over curves, J. Math. Soc. Japan, 30 (1978), 779-794.

[4] Harder, G. and Narashimhan, M. S., On the cohomology groups of moduli spaces of vector bundles on curves, Math. Ann., 212 (1975), 215-248.

[ 5 ] Horikawa, E., On deformations of quintic surfaces, Invent. Math., 31 (1975), 43-85. 
[6] Konno, K., Non-hyperelliptic fibrations of small genus and certain irregular canonical surfaces, Ann. Sc. Norm. Sup. Pisa. Ser. N, XX (1993), 575-595.

[ 7 ] Matsusaka, S., Some numerical invariants of hyperelliptic fibrations, J. Math. Kyoto Univ., 30 (1990), 33-57.

[ 8 ] Mukai, S. and Sakai, F., Maximal subbundles of vector bundles on a curve, Manuscripta Math., 52 (1985), 251-256.

[9] Nakayama, N., Zariski-decomposition problem for pseudo-effective divisors, In : Proceedings of the Meeting and the workshop "Algebraic Geometry and Hodge Theory", vol. I, Hokkaido Univ. Technical report series in Math. no. 16 (1990), 189-217.

[10] Reid, M., Surfaces of small degree, Math. Ann., 275 (1986), 71-80.

[11] Sun, X. T., Algebraic surfaces whose canonical image has a pencil of rational curves of degree two, Math. Z., 209 (1992), 67-74.

[12] Xiao, G., L'irrégularité des surfaces de type général dont le systéme canonique est composé d'un pinceau, Compositio Math., 56 (1985), 251-257.

[13] - Surfaces fibrées en courbes de genre deux, Lec. Notes in Math. 1137, Berlin, Heidelberg, New York, Springer, 1985.

[14] - Algebraic surfaces with high canonical degree, Math. Ann., 274 (1986), 473-483.

[15] - Fibred algebraic surfaces with low slope, Math. Ann., 276 (1987), 449-466.

[16] - Irregularity of surfaces with a linear pencil, Duke Math. J., 55 (1987), 597-602. 